\title{
Polo-like kinase 3 inhibits osteosarcoma cell proliferation and tumorigenesis via cooperative interaction with p21
}

\author{
HONGLIN LV ${ }^{1 *}$, GUANGTING GAO ${ }^{2 *}$, LINLIN ZHANG ${ }^{3}$ and YUJIE SUN ${ }^{1}$ \\ ${ }^{1}$ Department of Spinal Orthopedics, Yantai Yuhuangding Hospital Affiliated to Qingdao University Medical College, Yantai, \\ Shandong 264000; ${ }^{2}$ Department of Bone Surgery, Binzhou Central Hospital, Binzhou, Shandong 251700; \\ ${ }^{3}$ Department of Orthopedics, The People's Hospital of Gaomi, Gaomi, Shandong 261500, P.R. China
}

Received October 14, 2014; Accepted July 10, 2015

DOI: $10.3892 / \mathrm{mmr} .2015 .4304$

\begin{abstract}
Polo-like kinase 3 (Plk3) is a member of the Plk family. It is dysregulated in certain types of cancer, including colorectal and pancreatic cancer. However, the expression status and biological function of Plk3 in osteosarcoma (OS) remain poorly understood. Following evaluation of the role of Plk3 in OS, the present study indicates that Plk3 is downregulated in OS cell lines and tissues, and increased expression levels of Plk3 are associated with improved rates of overall survival of patients. In addition, to investigate the role of Plk3 in cell proliferation and tumorigenicity in vitro, two recombinant lentiviruses expressing Plk3 short hairpin RNA, as well as a recombinant plasmid carrying Plk3, were developed and transfected into Saos-2 and U2OS cells, respectively. Cell cycle analysis by flow cytometry demonstrated the influence of Plk3 on the arrest of cell cycle progression at the $G_{1}$ phase. Following knock down of Plk3, the growth and colony formation of Saos-2 cells increased significantly, whereas the overexpression of Plk3 resulted in the opposite trend. Furthermore, a 5-ethynyl-2'-deoxyuridine assay, using $\mathrm{U} 2 \mathrm{OS}$ cell lines, indicated the same tendency. The in vivo interaction between Plk3 and p21 in Saos-2 cells was detected and the protein level of p21 was observed to be consistent with that of Plk3. These results imply that Plk3 is involved in the inhibition of cell proliferation and tumorigenesis, which may occur via interactions with $\mathrm{p} 21$, thus, Plk3 may be considered as a potential candidate for targeted therapy of OS.
\end{abstract}

Correspondence to: Mr. Yujie Sun, Department of Spinal Orthopedics, Yantai Yuhuangding Hospital Affiliated to Qingdao University Medical College, 20 Yudong Road, Yantai, Shandong 264000, P.R. China

E-mail: yujiesu001@163.com

*Contributed equally

Key words: polo-like kinase 3, osteosarcoma proliferation, p21

\section{Introduction}

Osteosarcoma (OS) is considered to be one of the most frequent types of primary malignant bone tumor in children and adolescents (1). The underlying mechanisms of the formation and development of OS have been investigated for a long time. OS is a high-grade neoplasm with rapid growth and early metastasis (2), and lung metastasis is the predominant cause of OS-associated mortality. At present, high-dose chemotherapy and surgery are often adopted for the treatment of OS, however, this often results in resistance to chemotherapy, side effects and drug resistance, which influence therapeutic efficacy (3). Therefore, the priority is to identify more reliable markers for predicting treatment outcomes, therapeutic targets and effective therapeutic agents for suppressing OS metastasis.

As with other types of cancer, OS is often considered to be associated with the dysregulation of tumor suppressor genes and oncogenes, for example, p53 $(4,5)$ and retinoblastoma tumor suppressor (Rb) (6). Notably, p21 signaling is considered to be a pathway that regulates the progress of OS (7-9). The CDNK1A gene, which encodes p21(Cip1/Waf1), is a cyclin-dependent kinase inhibitor and is considered to be a major transcriptional target of the p53 protein (10). Various studies have demonstrated that $\mathrm{p} 21$ has an important role in the induction of normal and transformed cell differentiation (11-13). In tumor initiation, p21 suppresses the growth of malignant cells in vitro and in vivo (11-13) by inducing $\mathrm{G}_{1}$ - and $\mathrm{G}_{2} / \mathrm{M}$-phase cell cycle arrest (14-17). Furthermore, there is evidence that mice lacking p21 are predisposed to developing a wide spectrum of tumor types, which implies that p21 acts as a tumor suppressor (18). In a previous study, p21 was shown to act as an oncogene, although this was dependent on the type of culture (13). In addition, it has been identified that p21 depletion promotes sarcoma formation in mice (19) and numerous different clinical studies demonstrated in various types of cancer that the downregulation of p21 was associated with a poor prognosis (20-22). This indicates that p21 may act as a gene target, which regulates the response of tumor cells to chemotherapy and radiotherapy (23).

The mammalian polo-like kinase (Plk) family consists of five members: Plk1, Plk2, Plk3, Plk4 and Plk5 (24). It is a family of conserved protein serine/threonine kinases, which exhibit a polo-box domain (PBD) at the carboxyl-terminus $(25,26)$ 
(except for human Plk5, the kinase domain of which lacks kinase activity) (24-26). The founding member of the Plk family is Drosophila polo, which has regulatory involvement in mitosis and meiosis $(25,27)$. In addition, the biological functions of mammalian Plks are more diverse than those in lower eukaryotes (25). The focus of the present study is on $\mathrm{Plk} 3$, as recently various studies have indicated that Plk3 is a multifunctional protein that is involved in numerous different biological events, such as DNA damage responses, cell cycle control and apoptosis $(28,29)$. Plk3 shares high homology with Drosophila polo kinases, and possesses a kinase domain at the N-terminus (the function of which is phosphorylation of downstream serine/threonine proteins) and a Polo-box domain at the C-terminus (to bind interactive proteins). During cell cycle progression, marked changes occur regarding Plk3 abundance, kinase activity and subcellular distribution. A previous study demonstrated that Plk3 ablation in aged mice enhanced tumor angiogenesis (30). However, the function of Plk3 in OS remains poorly understood. The aim of the present study was to identify the role of Plk3 in osteosarcoma, to confirm the mechanism for Plk3 in facilitating tumorigenesis potential of osteosarcoma cells.

\section{Materials and methods}

Cell culture. The Saos-2, U2OS and HEK-293T human OS cell lines were obtained from the American Type Culture Collection (Manassas, VA, USA). The cells were cultured in Dulbecco's modified Eagle's medium (GE Healthcare Life Sciences, Logan, UT, USA) supplemented with $10 \%$ fetal bovine serum (GE Healthcare Life Sciences), 100 units of penicillin/streptomycin (Invitrogen Life Technologies, Carlsbad, CA, USA) and maintained at $37^{\circ} \mathrm{C}$ in air with a $5 \%$ $\mathrm{CO}_{2}$ humidified atmosphere.

Patients and specimens. The 30 OS samples and their adjacent healthy tissues were obtained from the surgical specimens of patients treated in Yuhuangding Hospital of Yantai (Yantai, China). Written informed consent was obtained from the patient. The samples were selected from patients with complete clinicopathologic information, who had been diagnosed and treated between June 2009 and November 2013. Of the 30 cases, there were 17 males and 13 females aged between 32 and 70 years (median, 55.3 years). The study was approved by the ethics committee of Qingdao University Medical College (Yantai, China). All patients were followed up for survival. The survival time was followed up from the diagnosis date up until cancer-associated death every 6 months. Only 2 patients were excluded from the study due to loss of contact, therefore these two cases were excluded from the statistical analysis $(n=28)$.

Reverse transcription-quantitative polymerase chain reaction (RT-qPCR). Total cellular RNA was isolated from the Saos- 2 cells using TRIzol reagent and amplified using an RT-qPCR kit (Invitrogen Life Technologies). The first strand of cDNA was synthesized using $1 \mu \mathrm{g}$ total RNA with M-MLV Reverse Transcriptase and the following primers were used for the detection of Plk3: Forward, 5-TCTGTTTGCCAA AGTTACCA-3 and reverse, 5-TTCTTCAAATCCATCTCC
ACTG-3. $\beta$-actin served as the internal control and was amplified using the following primers: Forward, 5-GTGGAC ATCCGCAAAGAC-3 and reverse, 5-AAAGGGTGTAAC GCAACTA-3. qPCR was conducted using an ABI PRISM ${ }^{\circledR}$ 7500 sequence detection system (Applied Biosystems Life Technologies, Foster City, CA, USA) and the relative expression levels of Plk3 in non-transduced and transduced Saos-2 cells were determined. The cycling conditions were as follows: $93^{\circ} \mathrm{C}$ for $2 \mathrm{mins},\left(93^{\circ} \mathrm{C}\right.$ for $1 \mathrm{~min}, 55^{\circ} \mathrm{C}$ for $1 \mathrm{~min}, 72^{\circ} \mathrm{C}$ for $1 \mathrm{~min}, 40$ cycles) $72^{\circ} \mathrm{C}$ for $10 \mathrm{~min}$, then held at $4^{\circ} \mathrm{C}$. A Plk3 plasmid was generated by inserting a full length of Plk3 into a pCMV-Tag2B vector (Invitrogen Life Technologies).

Western blot analysis. Non-transduced and transduced Saos-2 cells were lysed in $50 \mathrm{ml}$ lysis buffer $(100 \mathrm{mM}$ Tris- $\mathrm{HCl}$ (pH 7.4), 10 mM EDTA, 4\% SDS and 10\% glycine) on ice for $45 \mathrm{~min}$. The supernatants were collected by centrifugation at $12,000 \mathrm{x} \mathrm{g}$ for $30 \mathrm{~min}$ at $4^{\circ} \mathrm{C}$, and performance of a bicinchoninic acid method determined the protein concentration (BCA kit; GE Healthcare Life Sciences). Equal quantities (30 $\mu \mathrm{g}$ protein) of lysate were run on $10 \%$ SDS-PAGE gels (Invitrogen Life Technologies). Following electrophoresis, the protein blots were transferred onto nitrocellulose membranes (GE Healthcare Life Sciences) using electroblotting apparatus (Bio-Rad Mini-PROTEAN Tetra Electrophoresis system, Bio-Rad Laboratories, Inc., Hercules, CA, USA) and were then blocked with 5\% non-fat milk in Tris-buffered saline and Tween-20 (TBST) solution (Sigma-Aldrich, St. Louis, MO, USA). The membrane was incubated overnight with rabbit anti-Plk3 antibody (cat. no. SAB1300123), which was obtained from Sigma-Aldrich (dilution, 1:1,000) and rabbit anti-P21 antibody (cat. no. sc-397) obtained from Santa Cruz Biotechnology, Inc., Dallas, TX, USA; dilution, 1:500);. After three washes with TBST solution, the membrane was incubated with a secondary antibody (Santa Cruz Biotechnology, Inc.; dilution, 1:5,000) at room temperature for $60 \mathrm{~min}$. Immunoreactive bands were visualized using Western Blotting Luminol Reagent (Santa Cruz Biotechnology, Inc.) according to the manufacturer's instructions. The $\beta$-actin protein level served as a control (cat. no. sc-8432; mouse IgG1; Santa Cruz Biotechnology, Inc.).

The recombinant lentivirus and lentivirus vectors containing Plk3 short hairpin (sh)RNA\#1 (5-CCGGGC CCTTGCCTTTGTGGCCTTCCTCGAGGAAGGCCACAA AGGCAAGGGCTTTTTTG-3) or Plk3 shRNA\#2 (5-CCG GGATTTGAAGAAGGTCTGACTGCTCGAGCAGTCAGA CCTTCTTCAAATCTTTTTTG-3) were constructed and inserted into the PCMV-t green fluorescent protein (GFP) plasmid (Sigma-Aldrich). Non-silencing shRNA (5-CTA GCCCGGTTCTCCGAACGTGTCACGTATCTCGAGATA CGTGACACGTTCGGAGAATTTTTTTAAT-3) served as a control, as it does not target any genes in humans. Digestion analysis using a restriction endonuclease (Beijing TransGen Biotech Co., Ltd., Beijing China) confirmed the formation of the recombinant vector and all of the inserted sequences were verified by DNA sequencing. Lentiviruses were developed by triple transfection of $80 \%$ confluent 293T cells with Plk3 shRNA-expressing vector or the control shRNA, using Lipofectamine 2000 (Invitrogen Life Technologies). The cells were harvested in serum-free medium after 3 days and 


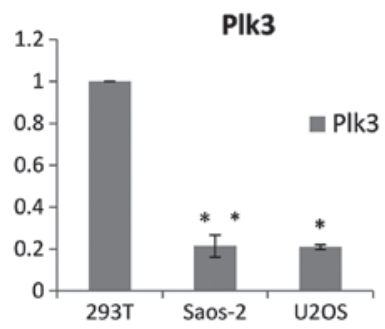

C

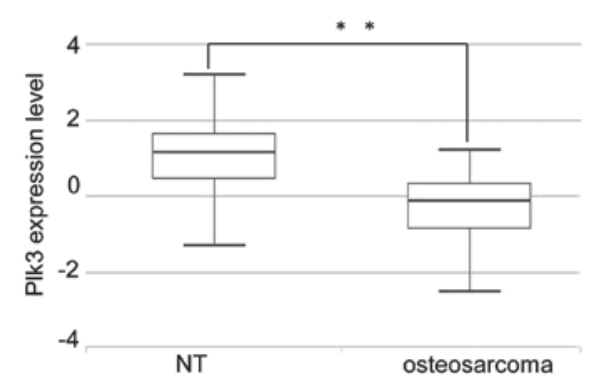

$\mathbf{E}$

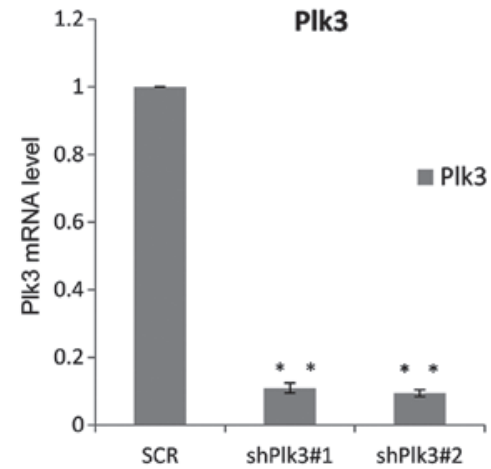

B

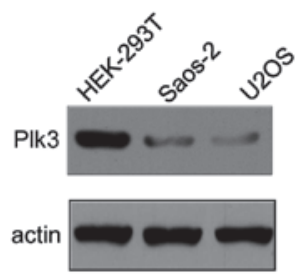

D

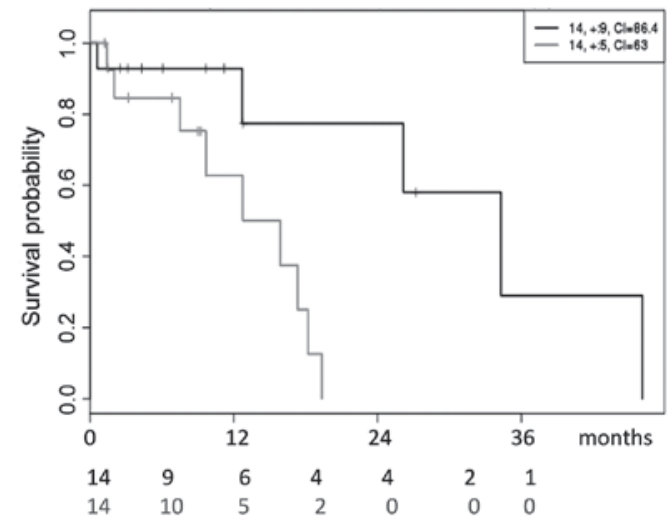

F

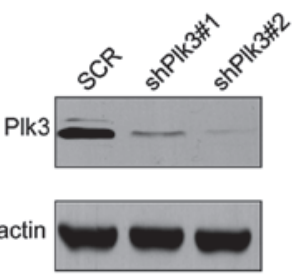

G

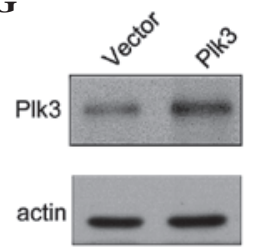

Figure 1. Abnormal expression of Plk3 in osteosarcoma tissue and cell lines. To determine the relative expression level of Plk3 in the human osteosarcoma cell lines, Saos-2 and U2OS, and human embryonic kidney cell line, HEK293T (A) qPCR [error bars represent average values \pm standard deviation (Student's t-test: ${ }^{*} \mathrm{P}<0.05$ and $\left.\left.{ }^{* *} \mathrm{P}<0.01\right)\right]$ and $(\mathrm{B})$ western blotting were conducted. The experiments were repeated three times and $\mathrm{n}=3$ for all experiments. (C) The expression of Plk3 was determined by RT-qPCR in 15 pairs of osteosarcoma and adjacent NT samples. U6 served as an internal control. The error bars represent the standard error of the mean (Student's t-test: $\left.{ }^{* *} \mathrm{P}<0.01\right)$. (D) The clinical data $(\mathrm{n}=28)$ were plotted using Kaplan-Meier survival curves and the 5-year survival rate was compared using the Cox log-rank test $\left({ }^{*} \mathrm{P}<0.05\right)$. The knockdown efficiencies of Plk3 were confirmed by $(\mathrm{E}) \mathrm{RT}-\mathrm{qPCR}$ and $(\mathrm{F})$ western blotting. $(\mathrm{G}) \mathrm{The}$ efficiency of Plk3 overexpression was detected by western blotting. Plk3, polo-like kinase 3; NT, non-cancerous tissue; RPPA, reverse phase protein array; RT-qPCR, reverse transcription-quantitative polymerase chain reaction; shRNA, short hairpin RNA; SCR.

filtered through a $0.45-\mu \mathrm{m}$ filter (Merck Millipore, Bedford, MA, USA).

Transduction was conducted by seeding Saos- 2 cells $\left(5 \times 10^{4}\right.$ cells/well) in 6 -well plates along with shRNA encoded recombinant lentiviruses against Plk3 (Lv-shPlk3) at a multiplicity of infection of 60 in serum-free growth medium. When serum-containing growth medium was added to the cells after $4 \mathrm{~h}$, there had been complete replacement of the growth medium with recombinant lentivirus. After 3 days of transfection, expression of the reporter gene, GFP was examined using fluorescence microscopy (Olympus BX53; Olympus Corporation).

Immunoprecipitation (IP). For IP assays, cells were washed with cold phosphate-buffered saline (PBS) and lysed with cold lysis buffer at $4^{\circ} \mathrm{C}$ for $45 \mathrm{~min}$. Whole cell lysates were incubated with Plk3 or P21 antibodies or normal rabbit/mouse immunoglobulin $\mathrm{G}$ on a rotator (Liuyi Instrument Factory, Beijing, China), overnight at $4^{\circ} \mathrm{C}$, followed by the addition of protein A/G Sepharose CL-4B beads (GE Healthcare Life Sciences) for $2 \mathrm{~h}$ at $4^{\circ} \mathrm{C}$. The beads were then washed five times with lysis buffer [50 mM Tris-Cl (pH 7.4), $150 \mathrm{mM}$ $\mathrm{NaCl}, 1$ mM EDTA, 1\% NP-40, 0.25\% sodium deoxycholate and protease inhibitor mixture]. The immune complexes were subjected to SDS-PAGE, followed by immunoblotting (IB) with secondary antibodies.

Flow cytometry. Saos-2 cells stably transfected with shPlk3 or control shRNA were synchronized by serum starvation for $24 \mathrm{~h}$, cells (density, >90\%) were then trypsinized (Sigma-Aldrich), collected, washed with PBS and fixed in $70 \%$ ethanol at $4^{\circ} \mathrm{C}$ overnight. After washing with PBS, cells were incubated with RNase A (Sigma-Aldrich) in PBS for $30 \mathrm{~min}$ at $37^{\circ} \mathrm{C}$ and stained with $50 \mathrm{mg} / \mathrm{ml}$ propidium iodide (Sigma-Aldrich). Cell cycle data were collected using a FACSCalibur ${ }^{\mathrm{TM}}$ flow cytometer (BD Biosciences, Franklin Lakes, NJ, USA) and analyzed with FlowJo 7.6.2 (FlowJo, LLC, Ashland, OR, USA), which was repeated three times. 
A
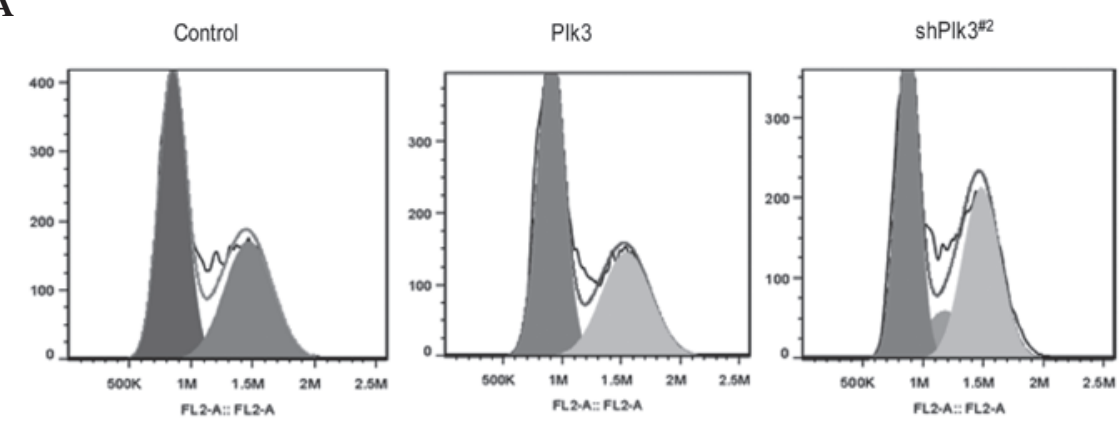

B

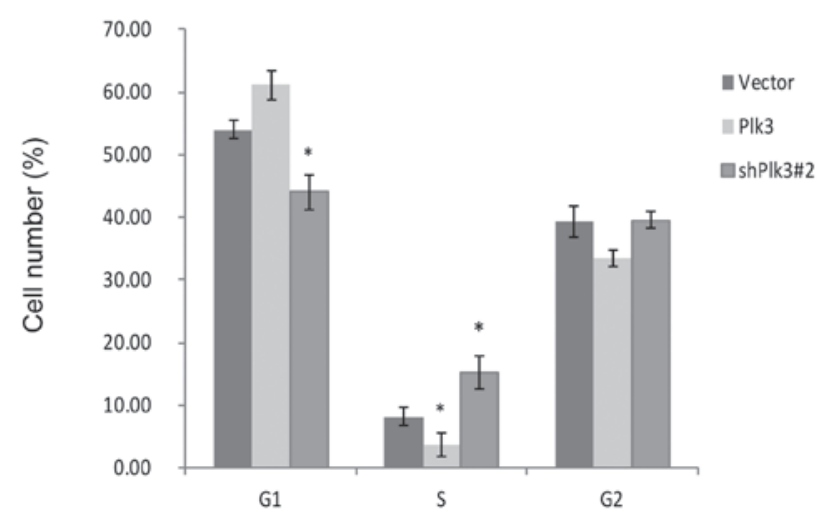

Figure 2. Plk3 modulated the cell cycle of the human osteosarcoma cells. (A) Cell cycle distribution of Plk3 was determined by flow cytometric analysis The $\mathrm{x}$ - and $\mathrm{y}$-axes show DNA content and cell number, respectively. The Saos-2 cells were transfected with vector (control), Plk3 or shPlk3\#2 and were synchronized by double thymidine block, and released into the cell cycle. (B) The data presented are representative of three independent experiments. Error bars represent the standard deviation of three independent experiments. " $\mathrm{P}<0.05$ vs. control. Plk3, polo-like kinase 3; shPlk3\#2, shRNA-Plk3\#2.

Colony formation assay. Following infection with the lentivirus for 3 days, a total of 1,000 Saos-2 cells were seeded into 6-well plates, and the medium was changed at 3-day intervals. G418 was used to select the cells transfected with the recombinant lentivirus and lentivirus vectors. After 10 days of culturing, the colonies formed were washed with PBS and fixed in $4 \%$ paraformaldehyde at $37^{\circ} \mathrm{C}$ for $30 \mathrm{~min}$, after which the colonies were stained with Coomassie (Sigma-Aldrich) for $15 \mathrm{~min}$, washed and air-dried. The colonies were counted under a microscope (Olympus BX46; Olympus Corporation). This experiment was performed in triplicate.

EdU assay. Saos-2 cells were seeded in 6-well plates for $24 \mathrm{~h}$ and transfected with the indicated shRNAs using Lipofectamine 2000. After $48 \mathrm{~h}$ of transfection, cells were incubated with EdU (Guangzhou Ribobio, Co., Ltd., Guangzhou, China) for $4 \mathrm{~h}$, fixed with $4 \%$ formaldehyde for $30 \mathrm{~min}$ and incubated with $2 \mathrm{mg} / \mathrm{ml}$ glycine for $5 \mathrm{~min}$. The assay was performed according to the manufacturer's instructions, using a fluorescence method. Furthermore, the positive and negative controls for the EdU assay were applied according to the manufacturer's instructions. Finally, 6-diamidino-2-phenylindole (Sigma-Aldrich) was used to stain the nuclei. The Olympus BX53 was used to observe the fluorescence.

Statistical analysis. SPSS version 17.0 was used for statistical analysis (SPSS, Inc., Chicago, IL, USA). Comparisons between cancer tissue and adjacent normal tissue were performed using a paired samples t-test. $\mathrm{P}<0.05$ was considered to indicate a statistically significant difference.

\section{Results}

Abnormal expression of Plk3 in the OS cell lines and tissues. The expression level of Plk3 was analyzed in different OS cell lines. As shown in Fig. 1A, downregulation of Plk3 was observed in the two examined OS cell lines, Saos-2 and U2OS, when compared with the HEK293 cells; the protein levels were also detected to be deceased (Fig. 1B). In addition, the expression levels of Plk3 were examined in 30 OS samples collected at the Yuhuangding Hospital of Yantai (Yantai, China) and the paired non-cancerous tissue (NT) samples were analyzed using RT-qPCR. The results demonstrate that the mean expression levels of Plk3 in the 30 OS samples (median, 1.4250; max., 3.1859; min., -1.3429) are significantly lower than those in the pairs of adjacent non-cancerous tissue (median, -0.1489; max., 1.2074; min., -2.5398; P=0; Fig. 1C). The expression level of Plk3 decreased in the OS samples, indicating that downregulation of Plk3 may be significant in OS. The OS samples obtained from the patients were divided into two groups, high and low, based on the median Plk3 expression level. Kaplan-Meier survival analyses of the different groups (Fig. 1D) demonstrated that higher Plk3 expression was associated with a longer overall survival time for patients with OS (hazard ratio=6.09; $\mathrm{P}=0.0239$ ).

shRNA inhibits the expression of Plk3 in OS cells. The efficiency of the recombinant lentivirus was measured by 
A

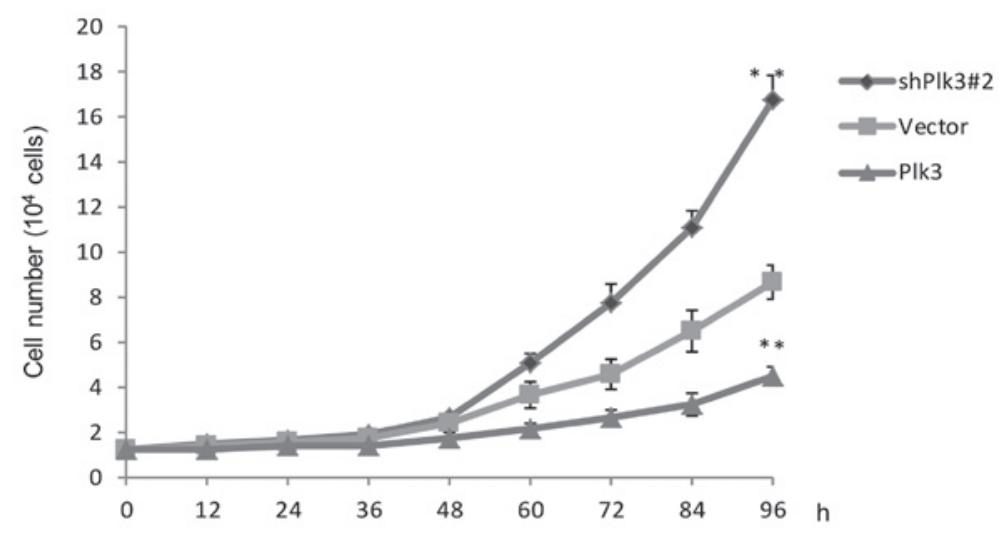

B
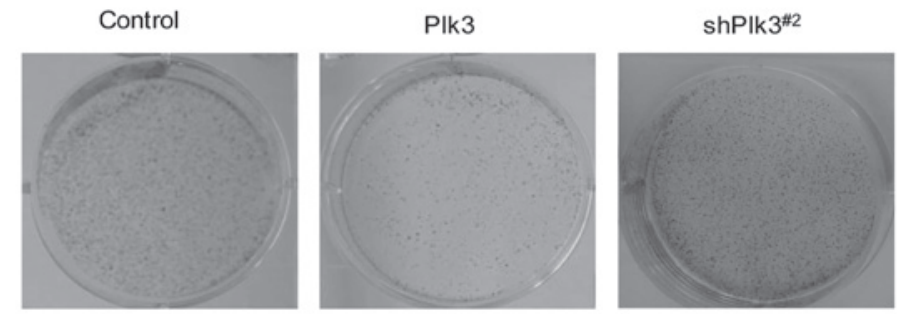

C

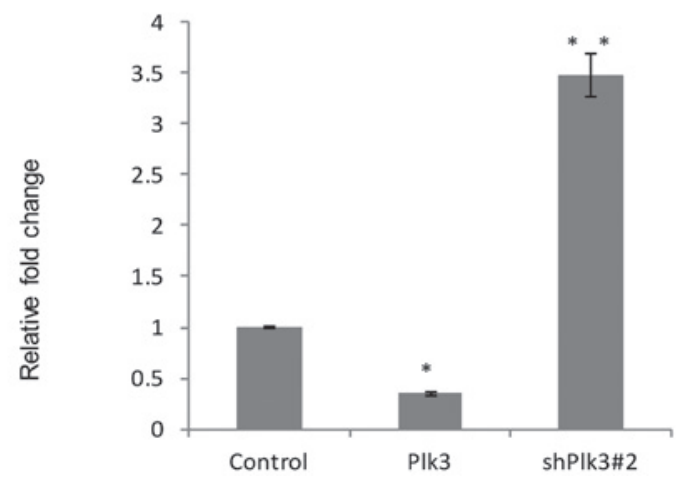

Figure 3. Plk3 modulated the proliferation and tumorigenicity of human osteosarcoma cells in vitro. (A) Plk3 inhibits cellular proliferation. Saos-2 cells were transfected with vector (control), Plk3 or shPlk3\#2 and subjected to growth curve analysis. (B) Equal numbers of cells were mixed and seeded into replicate plates. Plk3 suppresses the colony-forming efficiency of OS cells. Saos-2 cells transfected with vector (control), Plk3 or shPlk3\#2 were maintained in culture media for 14 days in the presence of $1 \mathrm{mg} / \mathrm{ml} \mathrm{G} 418$ prior to being stained with Coomassie. (C) Statistical analysis was conducted and the data are represented as the fold change over vector. Error bars represent the mean \pm standard deviation of triplicate measurements. ${ }^{*} \mathrm{P}<0.05$ and ${ }^{* *} \mathrm{P}<0.01$, vs. control. Plk3, polo-like kinase 3; shPlk3\#2, shRNA-Plk3\#2.

RT-qPCR and western blotting. The mRNA expression level of shRNA-Plk3\#1 was $\sim 10 \%$ that of the control group and shRNA-Plk3\#2 was $\sim 8 \%$ of the control $(\mathrm{P}<0.001$; Fig. 1E). The transfection ratio was observed to be $\sim 100 \%$. Western blot analysis demonstrated the protein levels (Fig. 1F) and indicated that the inhibitory ratios of shRNA-Plk3\#1 and shRNA-Plk3\#2 were 12.5 and $8.5 \%$, respectively. The efficiency of the Plk3 overexpression was also detected by western blotting (Fig. 1G).

Plk3 modulates the human OS cell cycle. To investigate the function of Plk3 in cell proliferation, the cell cycle distribution following transfection of shRNA-Plk3\#2, or the recombinant plasmid with Plk3 overexpression, was examined in Saos-2 cells by flow cytometry. Compared with mock-transfected cells, shPlk3-transfected cells revealed a substantial increase in $S$-phase populations and a decrease in $G_{1}$ phase populations, with Plk3 overexpression causing a block at the $G_{1}$ to $S$ phase
(Fig. 2A and B). These data indicate that inhibition of OS cell growth by Plk3 may be mediated via induction of $\mathrm{G}_{1}$ arrest.

Plk3 modulates the proliferation and tumorigenicity of human OS cells in vitro. In order to further understand the role of Plk3 in tumorigenicity, growth assays were performed in different cell vectors, Plk3 overexpression and Plk3 knock down. Saos-2 cells exhibiting Plk3 overexpression demonstrated marked growth inhibition, while the Plk3 knockdown vector (shPlk3\#2) revealed evident growth promotion, when compared with the untreated group (Fig. 3A). In addition, a colony formation assay was used (Fig. 3B). The average number of colonies in the control group was standardized to 1 . The data are presented as the fold change over vector for a better comparison. The number of colonies in the Plk3 overexpression group was found to be $35.3 \%$ compared with the control group. The shPlk3\#2 group was 3.48 fold greater than the control group. $(\mathrm{P}<0.05)$. The data indicated there was 
A
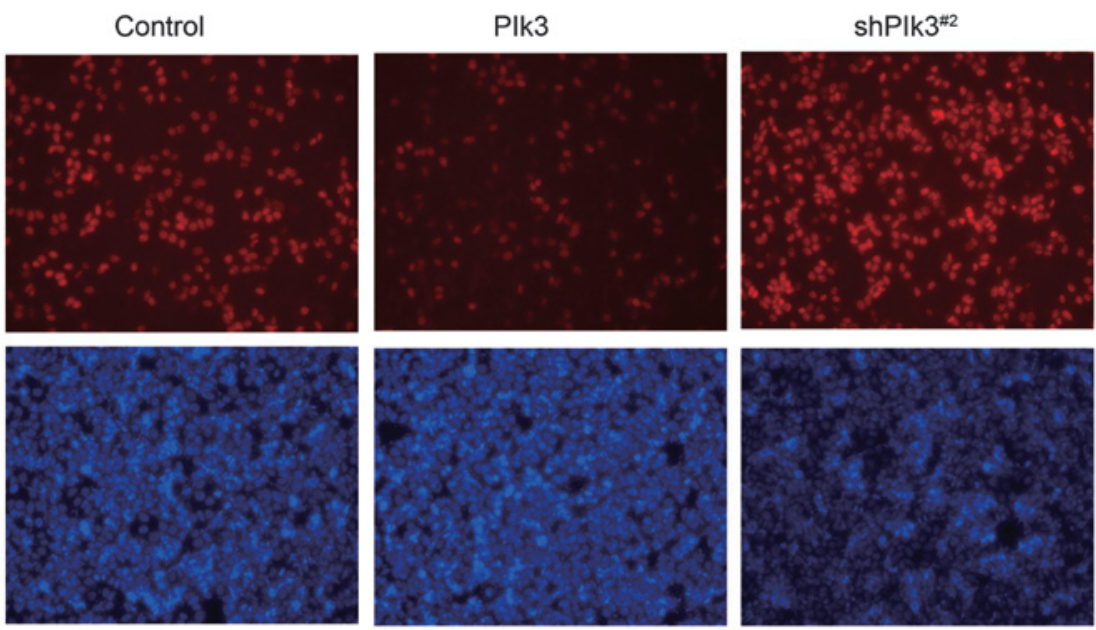

B

U2OS cell lines

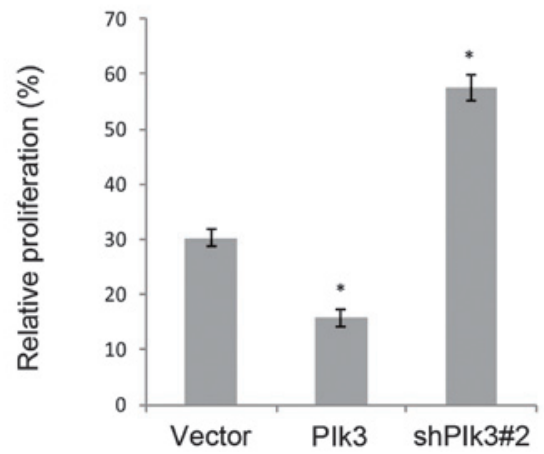

Figure 4. Performance of an EdU assay further demonstrated the inhibition of Plk3 on osteosarcoma cell proliferation. (A) U2OS cells were transfected with the indicated plasmids or shRNAs (shPlk3\#2). After $48 \mathrm{~h}$ of transfection, the EdU assay was performed using a fluorescence method. For each group, six different fields were randomly chosen and counted under a fluorescence microscope (magnification, x10). (B) Compared with control cells, the percentages of EdU-positive cells were counted. Error bars represent the mean \pm standard deviation for triplicate measurements. ${ }^{*} \mathrm{P}<0.05$ and ${ }^{* *} \mathrm{P}<0.01$, vs. vector. EdU, 5-ethynyl-2'-deoxyuridine; Plk3, polo-like kinase 3.

A
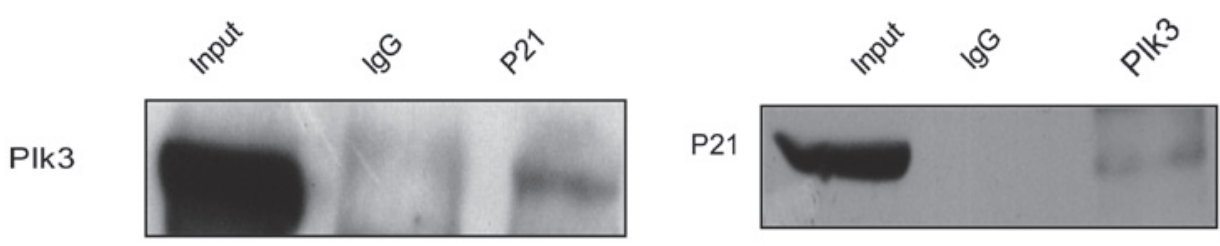

B
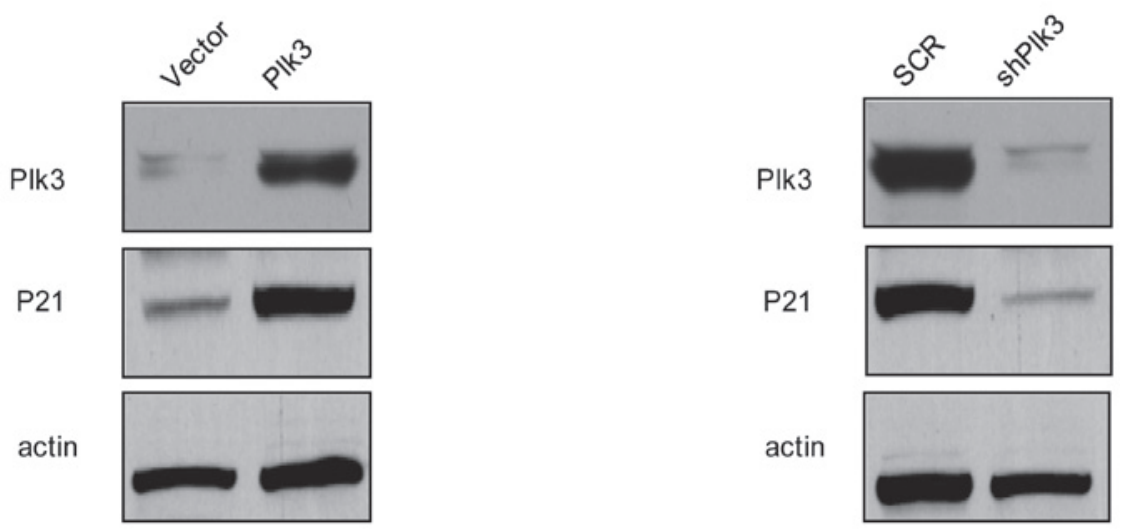

Figure 5. p21 is physically associated with Plk3, thus the protein level changes with Plk3. (A) Co-immunoprecipitation analysis of the association between Plk3 and $\mathrm{p} 21$. Whole cell lysates were immunoprecipitated with antibodies against the indicated proteins. IgG served as a negative control and whole lysates served as a positive control. Immunocomplexes were then immunoblotted using the indicated antibodies. (B) The protein level of p21 was detected by western blotting following Plk3 overexpression or knockdown with shRNAs (shPlk3\#2). Plk3, polo-like kinase 3; IgG, immunoglobulin G; SCR, scrambled control RNA. 
a significant increase in colony formation as a result of the low expression of Plk3 (Fig. 3C). An EdU assay using U2OS cell lines further demonstrated the function of Plk3 in proliferation, in vitro. When Plk3 was over expressed by the transfected recombinant plasmid, or knocked down by the shRNA, a similar tendency was detected (Fig. 4).

P21 is physically associated with Plk3 and the protein level varies with Plk3. A previous study demonstrated that Ect 2 promotes cell proliferation by regulating $\mathrm{p} 21$, a cell cycle-related gene, which is required for Rb protein-mediated $\mathrm{G}_{1}$ arrest (31). To further investigate the in vivo interaction between Plk3 and p21, total proteins from Saos-2 cells were extracted and co-IP experiments were performed. IP was performed with antibodies against $\mathrm{p} 21$ followed by IB with antibodies against Plk3 (Fig. 5A, left panel). Simultaneously, IP was performed with antibodies against Plk3 followed by IB with antibodies against p21 (Fig. 5A, right panel). The co-IP assay indicated that P21 was physically associated with $\mathrm{Plk} 3$ in vivo. To consider the role of $\mathrm{p} 21$ in OS, the effects of Plk3 on the expression levels of p21 were examined. The results revealed that protein levels of p21 increased in Saos-2 cells that had been transfected with Plk3 when compared with the control cells (Fig. 5B, left panel). When Plk3 was knocked down, the protein levels of p21 decreased (Fig. 5B, right panel). These results indicate that Plk3 interacts with p21 and the p21 protein level changes along with the Plk3 level, indicating that Plk3 may cause post-translational modification regulation of the $\mathrm{P} 21$ protein level and p21 activity may lead to cell cycle arrest.

\section{Discussion}

OS often originates in the metaphysis of long bones and has a high tendency for distant metastasis (32-34). Patients with metastasizing OS are often associated with a poor-prognosis signature, with $<20 \%$ mean 5 -year survival $(35,36)$. As a result, more detailed knowledge on the key mechanisms of the early stages of OS, as well as more reliable molecular markers for early diagnosis are urgently required. It is important to develop a more effective treatment strategy for OS patients with novel therapeutic agents that can target the tumor cells at an early stage (37).

In a previous study, data demonstrated that activated Plk3 leads to enhanced apoptosis in targeted cells; this effect was observed in cells that overexpress Plk3 (25). Additional evidence exists indicating that Plk3 ablation in mice does not noticeably perturb normal animal development (30). Therefore ,targeting Plk3 may result in fewer side effects in vivo.

In the present study, Plk3 was observed to be downregulated in OS tissue and cell lines, with higher Plk3 expression indicating improved overall survival. During the progress of OS, when Plk3 was knocked down with the appropriate shRNA, OS cell proliferation was enhanced; a similar phenomenon was also identified by observation of colony formation (using a soft agar assay) and by performance of an EdU assay. By contrast, overexpression of Plk3 reduced OS cell proliferation.

P21 is a cyclin-dependent kinase inhibitor, and a major transcriptional target of the p53 protein (10). As a tumor suppressor, it was proved, using mice models, that mice without the $\mathrm{p} 21(\mathrm{Cip} 1 / \mathrm{Waf} 1)$ protein are more sensitive to tumorigenesis (18). The absence of p21 protein enables the proliferation of cells with damaged DNA and promotes tumor progression (13). Notably, p21 is phosphorylated by various kinases, and its phosphorylation of serine or threonine residues (including Thr57, Thr145, Ser146 and Ser130) is considered to be particularly important. The exact roles of particular phosphorylation sites continue to be investigated. However, a previous study found that certain phosphorylation sites, such as at Thr57 (by mammalian sterile 20-like 1), at Thr145 and Ser146 (by AKT), or Ser130 (by p38 and c-Jun N-terminal kinase), increase the stability of the $\mathrm{p} 21$ protein (38). Additional studies have demonstrated that phosphorylation of p21 by AKT kinase leads to stabilization, as well as to distribution of p21 into the cytoplasm $(39,40)$.

In conclusion, the present results indicate that the dysregulation of Plk3 in OS affects cell proliferation in vitro. Furthermore, the function of Plk3 may depend on the interaction with $\mathrm{p} 21$, as the protein level of p21 varies with Plk3 expression. This may be due to the fact that $\mathrm{p} 21$ has numerous phosphorylation sites and Plk3 may phosphorylate the downstream proteins at the serine/threonine residues. However, the underlying mechanism requires further investigation.

\section{References}

1. Mirabello L, Troisi RJ and Savage SA: Osteosarcoma incidence and survival rates from 1973 to 2004: Data from the surveillance, epidemiology and end results program. Cancer 115: 1531-1543, 2009.

2. Marina N, Gebhardt M, Teot L and Gorlick R: Biology and therapeutic advances for pediatric osteosarcoma. Oncologist 9: 422-441, 2004.

3. Takeshita H, Gebhardt MC, Springfield DS, Kusuzaki K and Mankin HJ: Experimental models for the study of drug resistance in osteosarcoma: P-glycoprotein-positive, murine osteosarcoma cell lines. J Bone Joint Surg Am 78: 366-375, 1996.

4. Lin PP, Pandey MK, Jin F, Raymond AK, Akiyama $\mathrm{H}$ and Lozano G: Targeted mutation of p53 and Rb in mesenchymal cells of the limb bud produces sarcomas in mice. Carcinogenesis 30: 1789-1795, 2009.

5. Rubio R, Gutierrez-Aranda I, Sáez-Castillo AI, Labarga A, Rosu-Myles M, Gonzalez-Garcia S, Toribio Ml, Menendez P and Rodriguez R: The differentiation stage of $\mathrm{p} 53-\mathrm{Rb}$-deficient bone marrow mesenchymal stem cells imposes the phenotype of in vivo sarcoma development. Oncogene 32: 4970-4980, 2013.

6. Sosa-Garcia B, Gunduz V, Vázquez-Rivera V, Cress WD, Wright G, Bian H, Hinds PW and Santiago-Cardona PG: A role for the retinoblastoma protein as a regulator of mouse osteoblast cell adhesion: Implications for osteogenesis and osteosarcoma formation. PLoS One 5: e13954, 2010.

7. Shen H and Maki CG: p53 and p21(Waf1) are recruited to distinct PMl-containing nuclear foci in irradiated and Nutlin-3a-treated U2OS cells. J Cell Biochem 111: 1280-1290, 2010.

8. Lossaint G, Besnard E, Fisher D, Piette J and Dulic V: Chk1 is dispensable for $\mathrm{G} 2$ arrest in response to sustained DNA damage when the ATM/p53/p21 pathway is functional. Oncogene 30: 4261-4274, 2011.

9. Xu J, Yao Q, Hou Y, Xu M, Liu S, Yang L, Zhang L and Xu H: MiR-223/Ect2/p21 signaling regulates osteosarcoma cell cycle progression and proliferation. Biomed Pharmacother 67: 381-386, 2013.

10. Cmielová $\mathrm{J}$ and Rezáčová $\mathrm{M}$ : p21Cip1/Waf1 protein and its function based on a subcellular localization [corrected]. J Cell Biochem 112: 3502-3506, 2011.

11. Matsumoto T, Sowa Y, Ohtani-Fujita N, Tamaki T, Takenaka T, Kuribayashi K and Sakai T: p53-independent induction of WAF1/Cip1 is correlated with osteoblastic differentiation by vitamin D3. Cancer Lett 129: 61-68, 1998.

12. Gartel AL, Serfas MS and Tyner AL: p21-negative regulator of the cell cycle. Proc Soc Exp Biol Med 213: 138-149, 1996. 
13. Abbas T and Dutta A: p21 in cancer: Intricate networks and multiple activities. Nat Rev Cancer 9: 400-414, 2009.

14. Harper JW, Adami GR, Wei N, Keyomarsi K and Elledge SJ: The p21 Cdk-interacting protein Cip1 is a potent inhibitor of G1 cyclin-dependent kinases. Cell 75: 805-816, 1993.

15. Dulić V, Kaufmann WK, Wilson SJ, Tlsty TD, Lees E, Harper JW, Elledge SJ and Reed SI: p53-dependent inhibition of cyclin-dependent kinase activities in human fibroblasts during radiation-induced G1 arrest. Cell 76: 1013-1023, 1994.

16. Ando T, Kawabe T, Ohara H, Ducommun B, Itoh M and Okamoto T: Involvement of the interaction between $\mathrm{p} 21$ and proliferating cell nuclear antigen for the maintenance of G2/M rrest after DNA damage. J Biol Chem 276: 42971-42977, 2001.

17. Dash BC and El-Deiry WS: Phosphorylation of p21 in G2/M promotes cyclin B-Cdc2 kinase activity. Mol Cell Biol 25: 3364-3387, 2005.

18. Martin-Caballero J, Flores JM, Garcia-Palencia P and Serrano M: Tumor susceptibility of p21(Waf1/Cip1)-deficient mice. Cancer Res 61: 6234-6238, 2001.

19. Young NP, Crowley D and Jacks T: Uncoupling cancer mutations reveals critical timing of p53 loss in sarcomagenesis. Cancer Res 71: 4040-4047, 2011.

20. Liu X, Yang WT and Zheng PS: Msil promotes tumor growth and cell proliferation by targeting cell cycle checkpoint proteins p21, p27 and p53 in cervical carcinomas. Oncotarget 5: 10870-10885, 2014.

21. Luo DH, Zhou Q, Hu SK, et al: Differential expression of Notch1 intracellular domain and p21 proteins, and their clinical significance in gastric cancer. Oncol Lett 7: 471-478, 2014.

22. Zhang X, Liu J,Zang D, et al: Upregulation of miR-572 transcriptionally suppresses SOCS1 and p21 and contributes to human ovarian cancer progression. Oncotarget 6: 15180-15193, 2015.

23. Zhang Y, Sturgis EM, Zafereo ME, Wei Q and Li G: p14ARF genetic polymorphisms and susceptibility to second primary malignancy in patients with index squamous cell carcinoma of the head and neck. Cancer 117: 1227-1235, 2011.

24. Andrysik Z, Bernstein WZ, Deng L, Myer DL, Li YQ, Tischfield JA, Stambrook PJ and Bahassi el M: The novel mouse Polo-like kinase 5 responds to DNA damage and localizes in the nucleolus. Nucleic Acids Res 38: 2931-2943, 2010.

25. Dai W: Polo-like kinases, an introduction. Oncogene 24 : 214-216, 2005.

26. Lowery DM, Lim D and Yaffe MB: Structure and function of Polo-like kinases. Oncogene 24: 248-259, 2005.

27. Fenton B and Glover DM: A conserved mitotic kinase active at late anaphase-telophase in syncytial Drosophila embryos. Nature 363: 637-640, 1993.
28. Conn CW, Hennigan RF, Dai W, Sanchez Y and Stambrook PJ: Incomplete cytokinesis and induction of apoptosis by overexpression of the mammalian polo-like kinase, Plk3. Cancer Res 60: 6826-6831, 2000

29. Wang Q, Xie S, Chen J, Fukasawa K, Naik U, Traganos F, Darzynkiewicz Z, Jhanwar-Uniyal M and Dai W: Cell cycle arrest and apoptosis induced by human Polo-like kinase 3 is mediated through perturbation of microtubule integrity. Mol Cell Biol 22: 3450-3459, 2002

30. Yang Y, Bai J, Shen R, Brown SA, Komissarova E, Huang Y, Jiang N, Alberts GF, Costa M, Lu L, et al: Polo-like kinase 3 functions as a tumor suppressor and is a negative regulator of hypoxia-inducible factor-1 alpha under hypoxic conditions. Cancer Res 68: 4077-4085, 2008.

31. Iyoda M, Kasamatsu A, Ishigami T, Nakashima D, Endo-Sakamoto Y, Ogawara K, Shiiba M, Tanzawa H and Uzawa K: Epithelial cell transforming sequence 2 in human oral cancer. PLoS One 5: e14082, 2010.

32. Poletajew S, Fus L and Wasiutyński A: Current concepts on pathogenesis and biology of metastatic osteosarcoma tumors. Ortop Traumatol Rehabil 13: 537-545, 2011 (In English and Polish).

33. Endo-Munoz L, Evdokiou A and Saunders NA: The role of osteoclasts and tumour-associated macrophages in osteosarcoma metastasis. Biochim Biophys Acta 1826: 434-442, 2012.

34. Rainusso N, Wang LL and Yustein JT: The adolescent and young adult with cancer: State of the art-bone tumors. Curr Oncol Rep 15: 296-307, 2013.

35. Gorlick R and Meyers PA: Osteosarcoma necrosis following chemotherapy: Innate biology versus treatment-specific. J Pediatr Hematol Oncol 25: 840-841, 2003.

36. Hawkins DS and Arndt CA: Pattern of disease recurrence and prognostic factors in patients with osteosarcoma treated with contemporary chemotherapy. Cancer 98: 2447-2456, 2003.

37. Klein MJ and Siegal GP: Osteosarcoma: Anatomic and histologic variants. Am J Clin Pathol 125: 555-581, 2006.

38. Hwang CY, Lee C and Kwon KS: Extracellular signal-regulated kinase 2-dependent phosphorylation induces cytoplasmic localization and degradation of p21Cip1. Mol Cell Biol 29: 3379-3389, 2009.

39. Blagosklonny MV: Are p27 and p21 cytoplasmic oncoproteins? Cell Cycle 1: 391-393, 2002.

40. Sohn D, Essmann F, Schulze-Osthoff K and Jänicke RU: p21 blocks irradiation-induced apoptosis downstream of mitochondria by inhibition of cyclin-dependent kinase-mediated caspase-9 activation. Cancer Res 66: 11254-11262, 2006. 